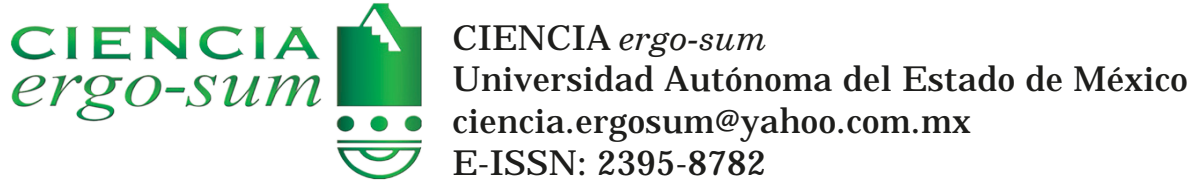

\title{
Turberas en Valle del Alto Mayo, Perú: importancia, amenazas y perspectivas de conservación
}

Quinteros-Gómez, Yakov Mario; Monroy-Vilchis, Octavio; Zarco-González, Martha Mariela Turberas en Valle del Alto Mayo, Perú: importancia, amenazas y perspectivas de conservación CIENCIA ergo-sum, vol. 28, núm. 1, marzo-junio 2021|e116

Universidad Autónoma del Estado de México, México

Esta obra está bajo una Licencia Creative Commons Atribución-NoComercial-SinDerivar 4.0 Internacional .

Quinteros-Gómez, Y. M., Monroy-Vilchis, O. y Zarco-González, M. M. (2021). Turberas en Valle del Alto Mayo, Perú: importancia, amenazas y per spectivas de conservación. CIE NCIA ergo-sum, 28(1). https://doi.org/10.30878/ ces.v28n1a10 


\title{
Turberas en Valle del Alto Mayo, Perú: importancia, amenazas y perspectivas de conservación
}

\section{Peatlands in the Alto Mayo Valley, Peru: Importance, threats and conservation perspectives}

\author{
Yakov Mario Quinteros-Gómez \\ Universidad Norbert Wiener, Perú \\ quinterosyakov@gmail.com \\ (D) https://orcid.org/0000-0003-2049-5971 \\ Octavio Monroy-Vilchis \\ Universidad Autónoma del Estado de México, México \\ omv@uaemex.mx, tavomonroyvilchis@gmail.com \\ (1) https://orcid.org/0000-0003-3159-6014 \\ Martha Mariella Zarco-González \\ Universidad Autónoma del Estado de México, México \\ martha.zarco.g@gmail.com \\ (D) https://orcid.org/0000-0003-4217-8272
}

Recepción: 17 de enero de 2020

Aprobación: 06 de mayo de 2020

\section{RESUMEN}

Los palmares de Mauritia flexuosa (aguajales) son considerados las principales turberas tropicales amazónicas que contribuyen al mantenimiento de la biodiversidad y del ciclo hidrológico, además de su aporte en el secuestro y almacenamiento de carbono. Mediante una revisión bibliográfica y observaciones de campo, se identifica la importancia, factores de amenaza y oportunidad para estos ecosistemas. Las turberas almacenan más del doble de carbono que todos los bosques del mundo en conjunto y conforman un grupo objetivo para el estudio del cambio climático. El acceso a sistemas de extracción no destructiva para la cosecha de los frutos debe estar articulado con planes socioeconómicos de desarrollo. Es imprescindible la aplicación de políticas participativas de educación ambiental e incentivos económicos a las comunidades que protegen estos territorios.

Palabras ClaVe: cambio climático, diversidad, Mauritia flexuosa, servicios ecosistémicos, turba.

\begin{abstract}
The Mauritia flexuosa palm swamp forest (aguajales) are considered the main Amazonian tropical peatlands that contribute to the maintenance of biodiversity and the hydrological cycle, in addition to their considerable contribution in carbon sequestration and storage. Through an exhaustive literature review and field observations we identify the importance, threat factors and opportunity for these ecosystems. The peatlands store more than twice as much carbon as all the world's forests together and form a target group for the study of climate change. Access to non-destructive extraction systems for the harvest of fruits must be articulated with socio-economic development plans. The application of participatory environmental education policies and economic incentives to the communities that protect these valuable territories is a priority.
\end{abstract}

Keywords: Climate change, diversity, Mauritia flexuosa, ecosystem services, peat.

\section{Aspectos generales de las turberas}

Las turberas son un tipo de humedal con suelo constituido por restos de vegetación acumulada parcialmente descompuesta. La descomposición total de la materia orgánica es inhibida por la ausencia de oxígeno en un 
ambiente inundado (figura 1) (Crump, 2017; COP, 2018). Por lo general, las turberas se encuentran ubicadas en depresiones entre dos ríos o canales, en suelos ácidos y con bajo contenido de nutrientes. En la Amazonia, estos escenarios favorecen el desarrollo y la expansión de especies vegetales adaptadas a estas condiciones por lo que son conocidas como especies hiperdominantes (Steege ter et al., 2013) que forman grandes extensiones de turberas cubiertas por bosques sucesionales sujetos a distintos periodos y niveles de inundación (Casanova y Brock, 2000).

El suelo de las turberas está conformado por dos capas: a) acrotelma: capa superior cuya profundidad es directamente proporcional a su impermeabilidad y proporcional de manera inversa a la tasa de descomposición orgánica. b) Catotelma: capa inferior con mayor profundidad, lenta descomposición y altamente saturada. Concentra la mayor cantidad de carbono y otros gases de efecto invernadero (GEI). Los suelos orgánicos de las turberas poseen alta concentración de partes vegetales en descomposición $(\geq 20 \%)$. Estos suelos oscuros y del tipo histoso son de lenta mineralización y descomposición bioquímica donde se forman sustancias húmicas manteniendo la capa superficial orgánica con saturación hídrica permanente (USDA, 2016).

A pesar de la existencia de diversos métodos para la evaluación y estudio de las turberas, la determinación de su profundidad es aún un obstáculo para estimar las reservas de carbono. En efecto, la dinámica sucesional de los bosques y la estacionalidad de las precipitaciones e inundaciones generan incertidumbre en la estimación de dichas reservas (Köchy et al., 2015).

Modelos de teledetección han estimado en 1.7 millones de $\mathrm{km}^{2}$ la superficie de las turberas tropicales (Gumbricht et al., 2017). Perú está considerado entre los 10 países megadiversos debido a que registra turberas desde la costa del pacífico hasta la cuenca amazónica, además pasa por la cadena montañosa de los Andes, valles interandinos y territorios transicionales como el piedemonte. Aunado a esto, es el noveno país con mayores extensiones de turberas con $49991 \mathrm{~km}^{2}$ (Crump, 2017).

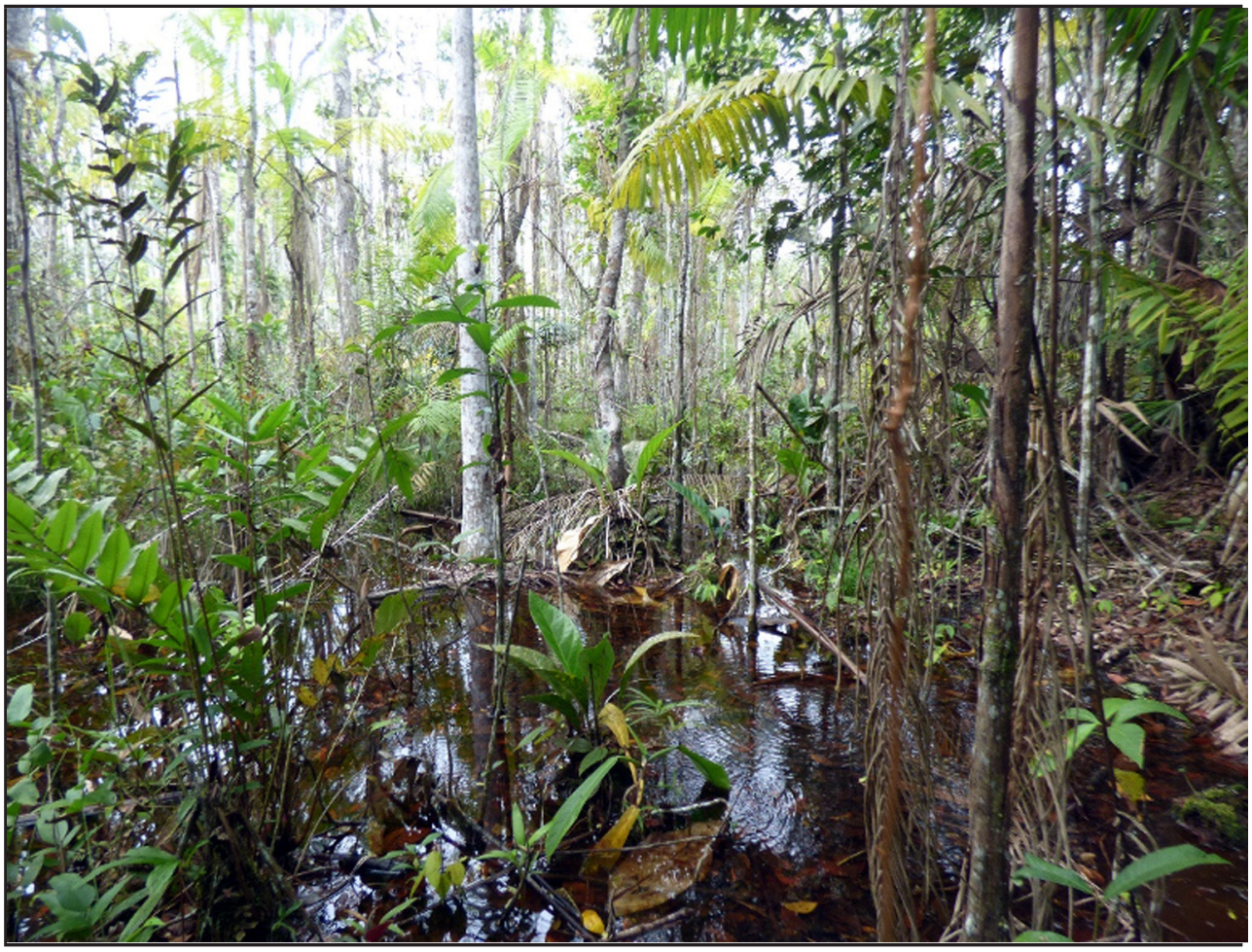

FIGURA 1

Pantano de turbera de palmeras (aguajal) permanentemente inundado en época de estiaje, Valle del Alto Mayo, Perú

Fuente: Dr. Yakov Quinteros. 
Si bien las turberas requieren condiciones de saturación hídrica permanente, el entorno paisajístico puede diferir entre ellas debido al nivel hídrico, evotranspiración y composición florística. En concreto, el tipo de vegetación produce distintos tipos de turba siendo imposible su reconocimiento en suelos oscuros, con presencia de humus, donde se encuentra muy descompuesta (USDA, 2016).

La formación de las turberas en la Amazonía ocurre en planicies de inundación y/o en depresiones que experimentan sucesivas inundaciones por desbordes estacionales del curso meándrico de los ríos. Finalizado el periodo de lluvias, el nivel de agua en la turbera disminuye sin llegar a secarse. Este proceso suele repetirse todos los años generando una lenta e incompleta descomposición de la materia orgánica y su acumulación en capas (figura 2) (Barthelmes et al., 2015). El proceso favorece el establecimiento y reclutamiento de especies adaptadas a estas condiciones, consideradas como hiperdominantes en la Amazonía (M. flexuosa, Socratea exhorriza, Ficus trigona, Coussapoa trinervia, Virola elongata, Symphonia globulifera, Inga stenoptera).

A partir de lo expuesto, se identifica la importancia, amenazas y perspectivas de conservación de las turberas en el Valle del Alto Mayo (VAM). Además, se destaca la relevancia y originalidad del manuscrito haciendo énfasis en la preservación de las turberas en el Valle del Alto Mayo.

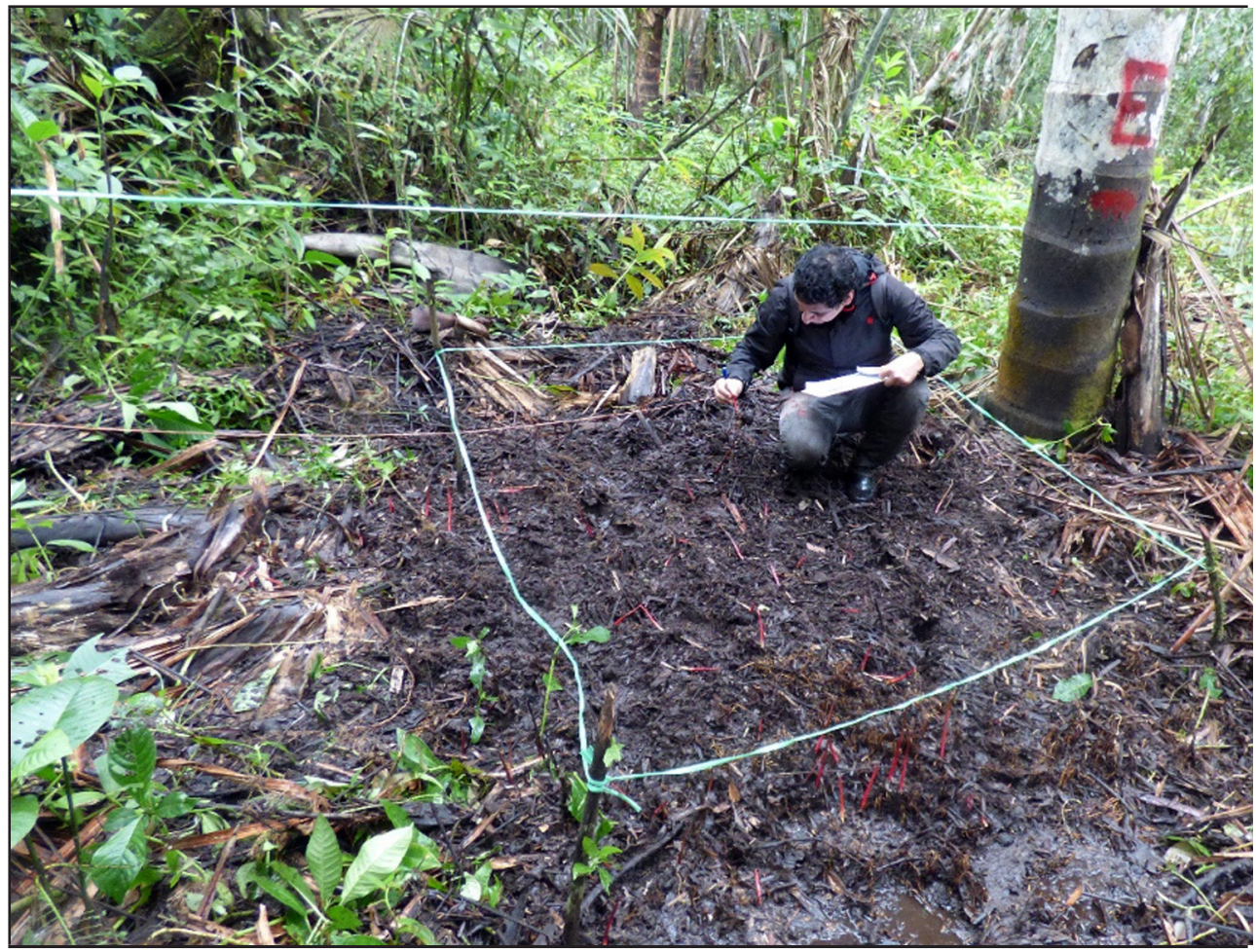

FIGURA 2

Suelo con materia orgánica parcialmente descompuesta (turba)

Fuente: Dr. Yakov Quinteros.

Nota: en rojo, al interior del cuadrante, se aprecian plúmulas de Mauritia flexuosa (germinación hipógea).

\section{Servicios ECOSISTÉmicos y LAS TURBERAS}

Los servicios ecosistémicos (SE) son definidos como los múltiples beneficios que la naturaleza aporta a la sociedad y que van en relación con mejorar la calidad de vida, la salud y la economía (MEA, 2005). Sin embargo, hasta llegar a la imposición de este concepto han sido muchos los cambios conceptuales y de paradigmas relacionados con las interacciones entre la sociedad, la naturaleza y el ambiente (Caro-Caro y Torres-Mora, 2015). El concepto de servicios ecosistémicos fue planteado en un inicio como naturaleza, pasando por recursos naturales y capital 
natural. A fines de los sesenta, ocurrió un cambio de paradigma hacia la perspectiva ecológica, donde se abordó el concepto en relación con los ecosistemas y funciones ecológicas que éstos desempeñan. Por último, desde 1992 con la Cumbre de la Tierra y su Convenio de Diversidad Biológica, se planteó conceptualmente como diversidad. Resultado de esta evolución conceptual (figura 3), los servicios ecosistémicos reúnen servicios culturales, de aprovisionamiento, de soporte, así como servicios de regulación (Wallace, 2007).

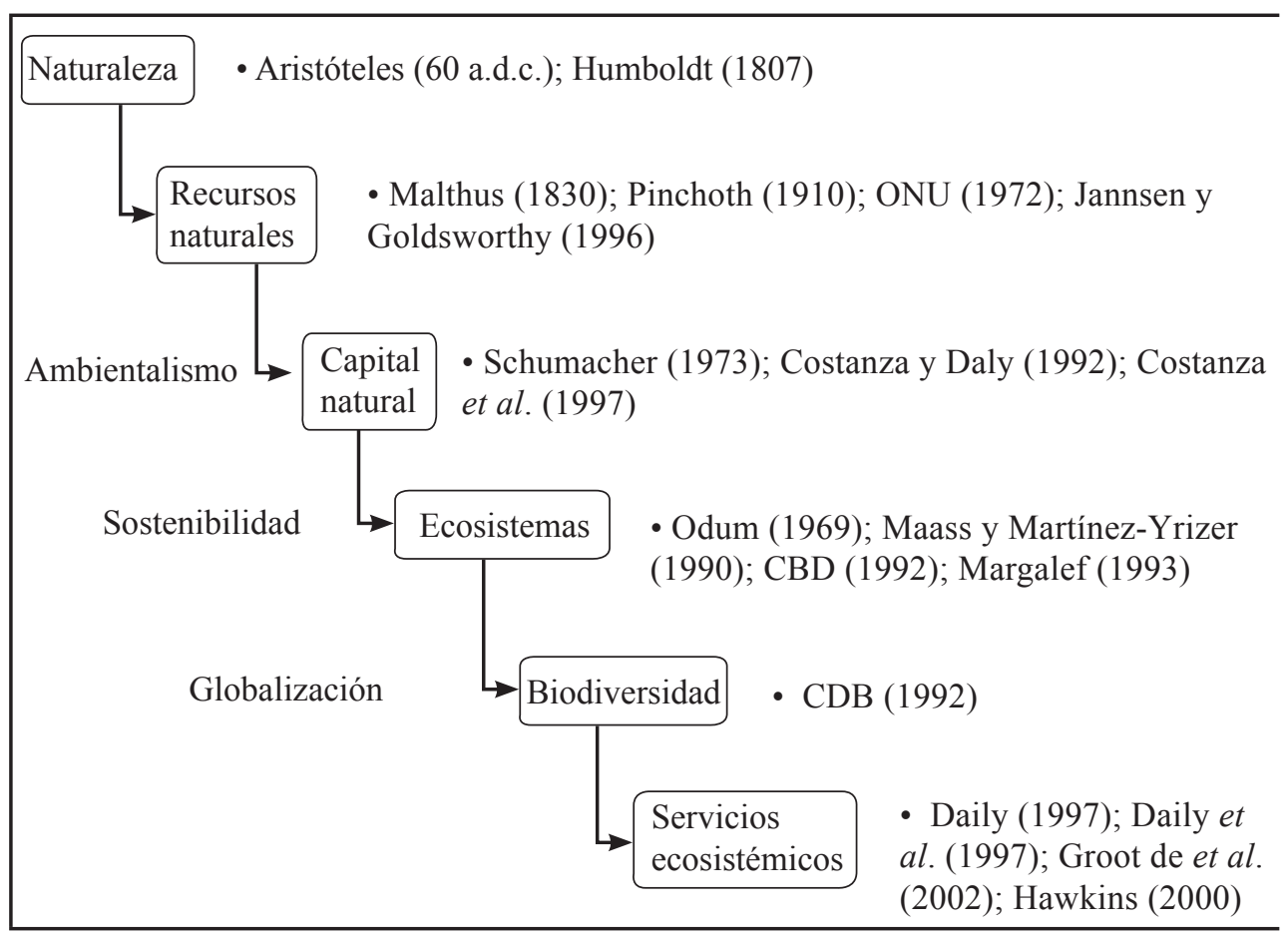

FIGURA 3

Evolución conceptual del enfoque servicios ecosistémicos

La acumulación de biomasa vegetal derivada de la fotosíntesis de las plantas es uno de los principales servicios ecosistémicos que prestan los bosques, especialmente las turberas. En este sentido, el dióxido de carbono $\left(\mathrm{CO}_{2}\right)$ atmosférico captado por la vegetación pasa a formar parte de la estructura forestal disminuyendo la cantidad de gases de efecto invernadero en el ambiente.

Las turberas intactas proporcionan una serie de servicios ecosistémicos. Son los principales sumideros de carbono, ya que almacenan más del doble de carbono que todos los bosques del mundo en conjunto (Barthelmes et al., 2015). El secuestro y almacenamiento de carbono en turberas se basa en niveles de agua consistentemente altos, por lo que también son importantes en la reserva, purificación y regulación de los ciclos del agua, además de la regulación del clima. Entre los servicios de aprovisionamiento que prestan las turberas se encuentran la provisión de alimentos, maderas, fibras y medicinas. En lo referente a soporte, se encuentran la formación de suelos, producción primaria y fotosíntesis. Por último, la educación ambiental, la diversidad cultural, así como la recreación y el ecoturismo representan los servicios culturales de las turberas (Wallace, 2007).

En el noroccidente de la región San Martín, en el Valle del Alto Mayo, se encuentran numerosas turberas de considerable extensión aisladas por efectos del crecimiento urbano poblacional y el incremento de la extensión de campos de cultivo. Estas turberas, localmente conocidas como aguajales, debido a la dominancia de $M$. flexuosa (aguaje), proporcionan importantes servicios ecosistémicos. En efecto, los aguajales son ecosistemas frágiles, considerados como las principales turberas tropicales naturales y se encuentran en toda la llanura aluvial de la cuenca amazónica, en grandes depresiones con deficiente drenaje. Sin embargo, también 
es posible encontrarlos en territorios de piedemonte, como es el caso de la región San Martín, un territorio de transición entre la llanura aluvial y los bosques montanos de la vertiente oriental, a una altitud entre los 800 y $1000 \mathrm{msnm}$, lo que los convierte en los agujales naturales a mayor elevación en Perú. En este sentido, las turberas del Valle del Alto Mayo son específicamente pantanos de turba de palmeras, es decir, turberas boscosas que se alimentan del agua de lluvia e inundaciones por desbordes estacionales de los ríos, un territorio con precipitación mayor a $1200 \mathrm{~mm} /$ año.

Debido a su importancia, los servicios ecosistémicos concentran cada vez más la atención de los investigadores, la prensa y la sociedad, lo cual propicia una sana discusión en el ambiente académico sobre las herramientas de gestión y políticas de manejo de estos humedales. Se hace imperativo que la política ambiental peruana sitúe tanto a los servicios ecosistémicos como a las turberas en el centro de la toma de decisiones de manera que permita contrarrestar y mitigar los efectos del cambio climático evitando riesgos potenciales para el ambiente y la sociedad en su conjunto.

\section{Biodiversidad en los aguajales del Valle del Alto Mayo}

Los aguajales en el Valle del Alto Mayo son característicos por su variedad de fisionomías forestales y su particular ubicación en depresiones de más de $20 \mathrm{~m}$ de profundidad compuestos por depósitos de turba cuaternaria (Alva et al., 1992) y cubiertos por bosques de 10-30 m de altura con abundancia de M. flexuosa. En estos territorios, las especies vegetales hiperdominantes son los recursos alimenticios más importantes para la fauna, ya que tienen una amplia distribución y fructifican de manera anacrónica (Khorsand et al., 2013) convirtiéndose en importantes elementos para el mantenimiento de aves, ungulados, roedores y primates (Silvius y Fragoso, 2002). Es decir, los aguajales concentran una importante fuente de biomasa y alimentos, además de otros servicios ecosistémicos, por lo que son considerados como ecosistemas estratégicos (Trujillo-González et al., 2011).

El anegamiento puede ser la causa de la variación en la composición, distribución, dinámica y abundancia de especies (Eiserhardt et al., 2011). Después de las inundaciones y la sedimentación, las plantas herbáceas (Costus), pequeños arbustos, enredaderas y árboles sucesionales se hacen visibles, en especial cerca de las orillas de los ríos, lo que contribuye a un aumento considerable en la extensión de los pantanos y ocasionalmente destruye la regeneración de especies leñosas (Kalliola et al., 1991) que favorece el reclutamiento de palmeras, lo que sugiere un proceso de sucesión hacia pantano de palmeras o aguajal (Hergoualc'h et al., 2017). Emilio et al. (2014) argumentan que las especies vegetales que aún persisten bajo estas condiciones han desarrollado adaptaciones como raíces aéreas (Ficus y Coussapoa), zancos (S. exorrhiza), bases del tronco aladas (Virola sp.) y neumatóforos que permiten el transporte de oxígeno en condiciones anaeróbicas ( $M$. flexuosa) (Melack y Hess, 2010).

La fragmentación, cambios en la cobertura vegetal por la extracción selectiva de especies y la caza de fauna nativa en pequeñas áreas (<20 ha) han favorecido la disminución de la abundancia y, en algunos casos, la extinción local de grandes vertebrados en los aguajales. Como consecuencia de ello, se han perdido las interacciones entre flora y fauna afectando de manera considerable los procesos de polinización, dispersión y depredación de frutos y semillas (Andreazzi et al., 2009), modificando la estructura del paisaje y formando pequeños remanentes forestales aislados (Fahrig, 2003).

\section{Importancia SOCIOCULTURAL DE MAURITIA FLEXUOSA}

M. flexuosa es la palmera con más amplia distribución de las especies restringidas a hábitats acuáticos en Sudamérica (Steege ter et al., 2013) debido al desarrollo de neumatóforos que le permiten sobrevivir a condiciones de inundación. Esto favorece la existencia de áreas casi exclusivas para esta especie que se distribuye desde el piedemonte oriental de los Andes hasta la costa atlántica en nueve países de la cuenca amazónica (Galeano et al., 2015). 
M. flexuosa es considerada como un importante recurso sociocultural y económico en los medios de subsistencia de las comunidades amazónicas (Endress et al., 2013). En efecto, todas las partes de la palmera son aprovechadas. Por ejemplo, las raíces de la palmera son utilizadas para curar enfermedades (Hoyos, 1992). El tallo o estípite de la palmera puede ser utilizado como soporte de las viviendas; si es abandonado en el bosque, ocurrirá su descomposición y será invadido por individuos de Rynchophorus palmarum (Coleóptera) que depositarán sus huevos. Al cabo de tres o cuatro meses se habrán desarrollado larvas conocidas localmente como suri, utilizadas en la alimentación y comercializadas por su alta demanda en los mercados amazónicos peruanos.

Las hojas de la palmera son utilizadas para el techado de viviendas u otras construcciones ligeras; en el bosque, llegan a formar parte de la turba juntamente con los restos de las infrutescencias (racimos). Los frutos se convierten en la parte más apreciada y demandada de la palmera y son comercializados bajo distintas formas (frutos maduros, pulpa, refrescos, helados, etc.) en las ciudades y pueblos amazónicos (Ponce et al., 2000).

En la cosmovisión de los pobladores rurales amazónicos tener plantas de aguaje en sus parcelas (jardines de las casas o áreas de cultivo) garantiza la presencia de agua. En palabras de los pobladores y trabajadores de las parcelas: "la palmera llama al agua, y ésta a su vez atrae a la fauna nativa", que es parte de la alimentación en las comunidades nativas (Chávez, 2014).

La cosecha de los frutos de aguaje en la región San Martín es parte de la problemática ambiental que aqueja a la especie, ya que hasta hace no más de 20 años las técnicas de recolección de los frutos consistían en una cosecha netamente destructiva donde se seleccionaban los mejores individuos (mayor cantidad de infrutescencias, infrutescencias más grandes, tamaño, color y calidad de los frutos), lo cual conducía así a una erosión genética de la especie.

Gracias al apoyo del gobierno regional y proyectos de educación ambiental, estos métodos de recolección han ido cambiando hacia prácticas sostenibles y amigables con la especie, donde acontece su revaloración en el ámbito regional (Quinteros-Gómez et al., 2016).

\section{Amenazas a los aguajales del Alto Mayo}

La región San Martín sufrió dos grandes olas migratorias durante el siglo pasado. La primera entre 1963 y 1968 y la segunda en 1975 con la construcción de la Carretera Marginal de la Selva (conecta la región amazónica con territorios altoandinos y costeros en el norte de Perú). Hasta aquellos años, la geografía de la región San Martín se caracterizaba por sus extensos aguajales e inmensos bosques de neblina a través del gradiente altitudinal. Sin embargo, este escenario se fue modificando en el transcurso de los años debido a políticas de intervención estatal que promovieron la ocupación de estos territorios, además de la exoneración de impuestos y el otorgamiento de subsidios para el cultivo de arroz y maíz. Esto provocó una acelerada deforestación de los territorios cercanos a los caminos y carreteras que mantienen aún inaccesibles áreas boscosas y alejadas. Los aguajales eran territorios poco aprovechados por los migrantes, ya que por la dificultad de acceso y a lo peligroso que resultaba adentrarse en un pantano eran considerados solamente como un territorio propicio para la caza de la fauna nativa. Sin embargo, la necesidad de suelos con deficiente drenaje para el cultivo de arroz fue el motivante para el inicio de la destrucción de los aguajales (figura 4). Actualmente, San Martín experimenta un crecimiento de $2 \%$ anual en la extensión del cultivo de arroz y concentra $27 \%$ de la producción nacional, lo cual representa en el Valle del Alto Mayo 41\% de la producción regional (Minagri, 2018).

En el periodo 2001-2015 se deforestaron al menos 228000 ha en la región San Martín (Ríos et al., 2018). Estos valores sugieren que los aguajales se encuentran en permanente amenaza por el cambio de uso de suelo. Sin embargo, esta no es la única problemática que enfrentan los aguajales; la extracción selectiva de especies maderables, ornamentales (orquídeas) y medicinales está creando descompensaciones en los ciclos biológicos de las especies, por lo que se desconoce la situación poblacional de muchas de ellas (Quinteros-Gómez et al., 2016), 
aunado a la pérdida de especies nativas y endémicas. La contaminación química de los suelos y el agua por el uso excesivo de agroquímicos, además de la deforestación y la exposición directa de la turba a la radiación solar, han ocasionado impactos irreparables en el paisaje, así como en la calidad de los servicios ambientales, lo cual representa un alto riesgo de incendio con la consecutiva liberación de miles de toneladas de carbono a la atmósfera como ha sucedido en turberas asiáticas que, por actividades antrópicas, liberaron grandes cantidades de carbono antiguo (almacenado durante miles de años) y registraron bajos niveles de COD (carbono orgánico disuelto), que sugiere la pérdida de turba almacenada durante miles de años (Evans, 2014).

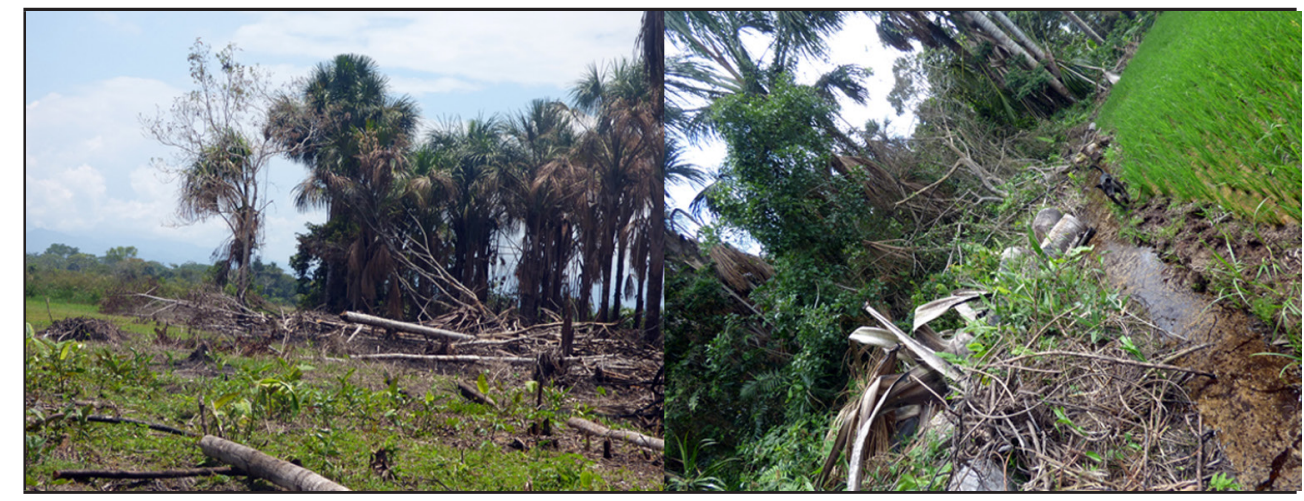

FIGURA 4

Destrucción de aguajales en el Valle del Alto Mayo por cambio de uso de suelo para cultivo de arroz Fuente: Dr. Yakov Quinteros.

\section{Acciones para la conservación de los aguajales}

Los aguajales son ecosistemas frágiles y sumamente vulnerables a los cambios en la dinámica natural del bosque, por lo que son considerados indicadores de la calidad ambiental y como grupo objetivo para el estudio del cambio climático. En este contexto, se están desarrollando esfuerzos tanto en el ámbito nacional como en el global. La primera acción que se está realizando en un marco nacional es el desarrollo de una guía técnica para definir turberas en Perú. Es una tarea encargada al Ministerio del Ambiente y en la cual se encuentran trabajando profesionales e investigadores en distintos tipos de turberas. Es la primera vez que se desarrolla este tipo de esfuerzo con el objetivo de calcular la extensión de las turberas en Perú. Terminada esta tarea se debería iniciar la fase de campo de manera que se pueda corroborar el tipo de turbera, su profundidad, vegetación dominante y reservas de carbono, entre otros aspectos.

Respecto a los aguajales del Valle del Alto Mayo, se están desarrollando estrategias para disminuir el impacto de la cosecha de frutos de aguaje permitiendo así que estén disponibles tanto en la palmera como en el suelo para que se pueda garantizar la disponibilidad del recurso para la fauna nativa, así como la germinación y patrones de reclutamiento de M. Alexuosa. Al mismo tiempo, en varios sectores del Valle del Alto Mayo las comunidades se han organizado (con el apoyo técnico de organizaciones no gubernamentales) y han creado asociaciones con fines de conservación. Estas asociaciones han solicitado y obtenido concesiones para el desarrollo de actividades sostenibles como el ecoturismo y el turismo rural. En efecto, la concesión obtenida por la ADECARAM (Asociación de Conservación de Aguajales y Renacales del Alto Mayo) se ha consolidado como modelo de gestión de la biodiversidad en Perú mediante un cambio de enfoque en el aprovechamiento de los recursos naturales pasando sus socios de la extracción de madera y la caza de especies nativas a promotores de la conservación de los aguajales. Además, han empezado a desarrollar alianzas estratégicas con investigadores y universidades con el fin de desarrollar investigaciones en flora y fauna a mediano y largo plazo. Este trabajo es un ejemplo de ello. 


\section{AnÁlisis PRospectivo}

El análisis prospectivo del uso de los aguajales por los distintos agentes (agricultores, dueños de parcelas, personal que trabaja en la extracción de los frutos) nos lleva a replantear la forma en que los recursos asociados a los aguajales están siendo aprovechados (tanto en la cuenca amazónica como en el Valle del Alto Mayo). En este sentido, los frutos de $M$. flexuosa son también utilizados en una aún incipiente industria cosmética debido a su alto contenido de tocoferoles y provitamina A: $7280 \mathrm{RE} / 100 \mathrm{~g}$ (De Rosso y Mercadante, 2007). Al mismo tiempo, se ha comprobado la presencia de genisteína (en la pulpa de aguaje), metabolito con propiedades estrogénicas para el tratamiento de malestares en la etapa menopaúsica y la regulación hormonal en las mujeres mayores de 40 años (Campos y Gutiérrez, 2013), además de actividad antioxidante por lo que es considerado un alimento funcional (Romero et al., 2015). Sin embargo, aún existen problemas en el procesamiento de los frutos de $M$. flexuosa, ya que se han reportado diferencias en las características físicas, composición de minerales de la pulpa, contenido de ácido ascórbico, compuestos fenólicos y polifenoles totales para frutos de M. flexuosa en poblaciones cercanas (Case et al., 2007; Vásquez-Ocmín et al., 2009), lo que demuestra la presencia de ecotipos a nivel de especie. En efecto, los ecotipos de M. flexuosa resultan ser limitantes para la industrialización de los frutos, por lo que la identificación de los mejores individuos y un manejo integrado de la especie representa un gran reto para esta nueva industria.

Aceites, harinas y extractos obtenidos de la pulpa de los frutos de M. Alexuosa, así como fibras de las hojas, tienen un futuro promisorio en el mercado de productos ecoamigables y sostenibles y también su comercialización asegurada por empresas socialmente responsables y reconocidas por su aporte ambiental (Barroso, 2008; Posada y Gómez, 2012).

Estos retos deben ser interiorizados y comprendidos por todos los actores involucrados en la cadena de comercialización de los frutos, así como por los agentes regionales y locales responsables de su conservación y tomadores de decisiones mediante la generación de políticas públicas de desarrollo rural sostenible para la especie. En este sentido, el acceso a sistemas de extracción no destructiva para la cosecha de los frutos debe ser una prioridad tan importante como la capacitación de "subidores" utilizando distintas técnicas de cosecha no destructiva potencializando sus habilidades e integrando estas actividades con planes socioeconómicos de desarrollo en los municipios de Moyobamba, Rioja, Pueblo Libre, Yuracyacu, Posic, Santa Elena y El Valle de la Conquista. Resulta también imprescindible el otorgamiento de subsidios por un periodo de hasta 10 años con el objetivo de estimular la agroindustria para el procesamiento de productos de aguaje que dinamicen la cadena productiva del fruto en conjunto con estrategias de mercado y marketing que lo destaquen como alimento funcional.

Como reflexión se deben considerar los pilares a futuro: el desarrollo agroindustrial sustentable de la especie, la educación ambiental y una adecuada gestión de los recursos en los aguajales de manera que las intervenciones en estos territorios estén enmarcadas en los principios de desarrollo sostenible y el principio precautorio del derecho ambiental; esto es, que cuando no se tenga certeza del nivel de vulneración o afectación de la actividad antrópica, es mejor no realizarla.

\section{Conclusiones}

Se identificaron los principales aspectos de las turberas relacionados con los servicios ecosistémicos y su conservación. En este sentido, el otorgamiento de concesiones de conservación y ecoturismo en el Valle del Alto Mayo ha permitido la recuperación y preservación de espacios donde aún se puede encontrar fauna nativa (potencial atractivo turístico). No obstante, ninguna estrategia ha logrado eliminar la problemática en la que se encuentran los aguajales por lo que deben redoblarse los esfuerzos para su aprovechamiento sostenible mediante el diseño de 
políticas participativas de educación, concientización y formación ambiental en todos sus niveles, al igual que el desarrollo de incentivos (mejora de los medios de subsistencia locales) para las comunidades que protegen estos valiosos territorios.

\section{REFERENCIAS}

Alva, J. E., Meneses, J. F., Chang, L. A., Lara, J. L. y Nishimura, T. (1992). Efectos en el terreno ocasionados por los sismos del Alto Mayo en Perú. Proceedings of the Tenth World Conference on Earthquake Engineering. Balkema, Rotterdam.

Andreazzi, C. S., Pires, A. S. y Fernandez, F. (2009). Mamíferos e palmeiras neotropicais: interações em paisagens fragmentadas. Oecologia Brasiliensis, 13(4), 554-574.

Barroso, F. (2008). La responsabilidad social empresarial. Un estudio en cuarenta empresas de la ciudad de Mérida, Yucatán. Contaduría y Administración, 226, 73-91.

Barthelmes, A., Ballhorn, U., \& Couwenberg, J. (2015). Consulting Study 5: Practical guidance on locating and delineating peatlands and other organic soils in the Tropics. The High Carbon Stock Science Study 2015.

Campos, C. K. y Gutiérrez, L. C. (2013). Actividad estrogénica del extracto hidroalcohólico del fruto de aguaje Mauritia flexuosa L.f. en ratas ovariectomizadas. Revista Peruana de Investigación Materna Perinatal, 13(2), 14-8.

Caro-Caro, C. y Torres-Mora, M. A. (2015). Servicios ecosistémicos como soporte para la gestión de sistemas socioecológicos: aplicación en agroecosistemas. Orinoquia, 19(2), 237-252.

Casanova, M. T., \& Brock, M. A. (2000). How do depth, duration and frequency of flooding influence the establishment of wetland plant communities? Plant Ecology, 147, 237-250.

Case, C., Lares, M., Palma, A., Brito, S., \& Pérez, E. (2007). Blood glucose and serum lipid levels in the Venezuelan Warao tribe: Possible relationship with moriche fruit (Mauritia flexuosa L.) intake. Nutrition, Metabolism \& Cardiovascular Diseases, 17, 1-2.

Chávez, A. (2014). Situación actual de una plantación de Mauritia flexuosa "aguaje" L.f. en suelos de restinga baja en Puerto Almendras, Loreto. Universidad Nacional de la Amazonía Peruana.

COP (Conferencia de las Partes Contratantes en la Convención de Ramsar sobre los Humedales). (2018). Resolución XIII. Orientaciones para identificar turberas como humedales de importancia internacional (sitios Ramsar) para la regulación del cambio climático mundial como argumento adicional a los criterios existentes de Ramsar. Disponible en https://www.ramsar.org/sites/default/files/documents/library/xiii.12 identifying_peatlands_ramsar_sites_s.pdf

Crump, J. (2017). Smoke on Water-Countering global threats from peatland loss and degradation. A UNEP Rapid Response Assessment. United Nations Environment Programme and GRID-Arendal, Nairobi and Arendal. Retrieved from https:// http://www.grida.no/publications/355

De Rosso, V. V., \& Mercadante, A. Z. (2007). Identification and quantification of carotenoids, by HPLC-PDAMS/MS, from Amazonian Fruits. Journal of Agricultural and Food Chemestry, 55(13), 5062 -5072

Eiserhardt, W. L., Svenning, J. C., Kissling, W. D., \& Balslev, H. (2011). Geographical ecology of the palms (Arecaceae): Determinants of diversity and distributions across spatial scales. Annals of Botany, 108, 1391-1416.

Emilio, T., Quesada, C. A., Costa, F. R. C., Magnusson, W. E., Schietti, J., Feldpausch, T. R., Brienen, R. J. W., Baker, T. R., ... Phillips, O. L. (2014). Soil physical conditions limit palm and tree basal area in Amazonian forests. Plant Ecology and Diversity, 7, 215-229. 
Endress, B. A., Horn, C. M., \& Gilmore, M. P. (2013). Mauritia flexuosa palm swamps: Composition, structure and implications for conservation and management. Forest Ecology Management, 302, 346-353.

Evans, C. D., Page, S. E., Jones, T., Moore, S., Gauci, V., Laiho, R., Hruska, J., Allott, T. E. H., Billett, M. F., Tipping, E., Freeman, C., \& Garnett, M. H. (2014). Contrasting vulnerability of drained tropical and high-latitude peatlands to fluvial loss of stored carbon. Global Biogeochemical Cycles, 28,1215-1234.

Fahrig, L. (2003). Effects of habitat fragmentation on biodiversity. Annual Review of Ecology, Evolution, and Systematics, 34, 487-515. https:// doi.org/10.1146/annurev.ecolsys.34.011802.132419

Galeano, A., Urrego L. E., Sánchez, M., \& Peñuela, M. C. (2015). Environmental drivers for regeneration of Mauritia flexuosa L.f. in Colombian Amazon swamp forest. Aquatic Botany, 123, 47-53.

Gumbricht, T., Roman-Cuesta, R. M., Verchot, L., Herold, M., Wittmann, F., Householder, E., \& Murdiyarso, D. (2017). An expert system model for mapping tropical wetlands and peatlands reveals South America as the largest contributor. Global Change Biology, 23, 3581-3599.

Hergoualc’h, K., Gutiérrez-Vélez, V. H., Menton, M. y Verchot, L. V. (2017). Caracterización de la degradación de los pantanos de palmeras turbosos desde el espacio y sobre el terreno: un estudio exploratorio en la Amazonia peruana. Documentos Ocasionales 179. Bogor: CIFOR. Disponible en http://www.cifor.org/ publications/pdf_files/OccPapers/OP-179.pdf

Hoyos, F. J. (1992). Palma moriche (Mauritia flexuosa L.f.). Natura, 94, 18-23

Köchy, M., Hiederer, R., \& Freibauer, A. (2015). Global distribution of soil organic carbon-Part 1: Masses and frequency distributions of SOC stocks for the tropics, permafrost regions, wetlands, and the world. SOIL, $1,351-365$.

Kalliola, R., Puhakka, M., Salo, J., Tuomisto, H., \& Ruokolainen, K. (1991). The dynamics, distribution and classification of swamp vegetation in Peruvian Amazonia. Annales Botanici Fennici, 28, 225-239.

Khorsand, R. R., Barbosa, R. I., \& Koptur, S. (2013). How do habitat and climate variation affect phenology of the Amazonian palm, Mauritia flexuosa? Journal of Tropical Ecology, 29(3), 255-259.

Melack, J. M., \& Hess, L. L. (2010). Remote sensing of the distribution and extent of wetlands in the Amazon basin. In W. J. Junk, M. T. F. Piedade, F. Wittmann, J. Schöngart, J., \& P. Parolin (Eds.), Amazonian floodplain forests (pp. 43-59). Springer.

MEA (Millennium Ecosystem Assessment). (2005). Ecosystems and Human Well-being: Biodiversity Synthesis. World Resources Institute. Washington, DC.

Minagri (Ministerio de Agricultura y Riego). (2018). Informe de Coyuntura Arroz 2001-2017. Disponible en http://siea.minagri.gob.pe/siea/sites/default/files/Informe-coyuntura-arroz-280818_0.pdf

Ponce, M. E., Stauffer, F., Olivo, M. L. y Ponce, M. (2000). Mauritia flexuosa L.f. (Arecaceae) una revisión de su utilidad y estado de conservación en la cuenca amazónica con especial énfasis en Venezuela. Acta Botánica Venezuelica, 23,19-46.

Quinteros-Gómez, Y. M., Roca-Alcázar, F. y Quinteros-Sánchez, V. (2016). Ecología, uso y conservación de los aguajales en el Alto Mayo, San Martín. Un estudio sobre las concentraciones de Mauritia flexuosa en la selva peruana, en C. Lasso, G. Colonnello, M. Moraes (Eds.), Morichales, cananguchales y otros palmares inundables de Suramérica Parte II (pp. 265-283).

Posada, S. y Gómez, R. B. (2012). Posibilidades de penetración al mercado colombiano con alimentos funcionales. Escuela de Ingeniería de Antioquia.

Ríos, S., Liza, R., Smith, R. Ch. y Montes, M. R. (2018). Deforestación en el noroeste de la Amazonía peruana. Disponible en https://www.iwgia.org/images/documentos /deforestacin.pdf 
Romero, A. B., Martins, M. D., Nunes, P. H., Ferreira, N. R., Brito, A. K., Cunha, P. F., Lima, A. D., Assis, R. C. \& Araújo, E. M. (2015). In vitro and in vivo antioxidant activity of buriti fruit (Mauritia flexuosa L.f.). Nutrición Hospitalaria, 32(5), 2153-61.

Silvius, K. M., \& Fragoso, J. M. V. (2002). Pulp handling by vertebrate seed dispersers increases palm seed predation by bruchid beetles in the northern Amazon. Journal of Ecology, 90, 1024-1032.

Steege ter, H., Nigel C. A., Pitman, Sabatier, D., Baraloto, C., P. Salomão, Guevara, J. E., ... R. Silman, M. (2013). Hyperdominance in the Amazonian Tree Flora. Science, 342, 1243092. Retrieved from https://science. sciencemag.org/content/342/6156/1243092

Trujillo-González, J. M., Torres, M. A. M. y Santana, E. C. (2011). La palma de Moriche (Mauritia flexuosa L.f.) un ecosistema estratégico. Orinoquia, 15, 62-70.

USDA (U. S. Department of Agriculture). (2016). Vasilas, L. M., Hurt, G. W. \& Berkowitz, J. F. (Eds.), Field indicators of hydric soils in the United States. Retrieved from https://www.nrcs.usda.gov/ Internet/FSE_DOCUMENTS/nrcs142p2_053171.pdf.

Vásquez-Ocmín, P., Sotero, V., Del Castillo, D., Freitas, L. y Maco, M. (2009). Diferenciación química de tres morfotipos de Mauritia flexuosa L.f. de la Amazonía peruana. Revista Sociedad Química del Perú, 75(3), 320-328.

Wallace, K. J. (2007). Classification of ecosystem services: Problems and solutions. Biological Conservation, 139, 235-246.

\section{BY-NC-ND}

